\title{
Ueber den Grund der accommodatorischen Formveränderung der Linse.
}

\author{
Von
}

Medicinalrath Witter in Meiningen.

Zwci Flüssigkeiten A und B, welche durch eine bewegliche Scheidewand getrenut, ein geschlossenes Gefäss erfüllen, stehen abgesehen von den Wirkungen der Schwere und der Molekularkräfte, unter allen Umständen unter gleichom Druck. Jirfährt die Flüssigkeit $\Lambda$ den I) ruck $k$, so theilt sich dieser der Flüssigkeit B gerade so mit, als ob die Scheidewand nicht vorhanden wäre. Büsst aber die Scheidewand ihre passive Beweglichkeit cin und übt sie durch eigene Kraftentwicklung von sich ans den Gegendruck nk auf A, so wird die Vertheilung des Iruckes eine ungleiche in der Weise, dass A nunmehr unter einen Druck zu stehen kommt, welcher der Summe $k(1+n), B$ unter einen solchen, welcher dem Unterschiede k $\left(\begin{array}{lll}1 & \ldots & \mathrm{n}\end{array}\right)$ der beiden drückenden Kräfte gleichkommt. 
Derselbe Fall tritt ein, wenn eine anfangs erschlaffte Membritn, welche die Stelle der beweglichen Scheidewand vertritt, durch irgend eine Kraft in Spannung versetzt wird, und zugleich das Bestreben erhält, den Raum der Flüssigkeit, von welcher die Drucksteigerung ausgeht, zu vermindern, sie also einen aktiven Gegendruck auf diese ausübt.

Im Auge liegt der Fall so, dass die Kammerflïssigkeit einem permanenten physiologischen Drucke unterlicgt, die Flüssigkeit des Petit'schen Kanales dagegen nicht. Beide sind durch die Zonula getrennt, welche durch einen eigenen Muskel abwechselnd in Erschlaffung und in Spannung versetzt werden kann, wodurch im ersten Falle der Druck des Kammerwassers auf den Inhalt des Petit'schen Kanals ungeschwächt fortgepflanzt wird, im zweiten das Kammerwasser eine der Muskelwirkung entsprechende Zunahme, die den I'etit'schen Kanal urfüllende Flüssigkeit eine gleiche Verminderung des Drucks erfährt. In ersten Falle ist die Linse ringsum einem gleichmässigen Drucke anusgesetzt, im zweiten wird sie von den Polen her, sowcit sie mit dem Kammerwasser und dem Glaskörper in Bcrïhrung steht, stärker gedrückt, als vom Rande her, so weit der Petit'sche Kanal reicht, sie erleidet also eine Zusammendrückung in diesem Sinne oder eine Vorkürzung der Achse, welche wieder einer Pormveründerung in entgegengesctztem Sinne weicht, sobald die Spannming der Zonula nachlässt.

Um eine annähernd richtige Vorstellung von der Grösse dieser Druckschwaukungen zu erhalten, nach welcher man ihre Wirkungen bernesseu kann, substituirt man der Linse eine ihrer Obertläche congruente mathematische feste Fläche, deren Theile den einwirkenden Kräften gegenüber unzertrennlich verbunden sind, so dass ein in Iezug auf dic I'ole symmetrisch vertheilter 
Druck in der Richtung der Achse nur eine Verkürzung dieser und eine Erweiterung des Aequators, ein Druck senkrecht auf die Achse nur eine Verlängerung derselben und Abnahme des Durchmessers des Aequators unter entsprechender Anordnung der zwischenliegenden Punkte bewirken kann, und zwar unter Ausschluss einer jedeu anderen Richtung der Bewegung der cinzelnen Theile. Den Druck, welcher irgend einen Punkt dieser Fläche trifft, zerlegt man in zwei Componenten, deren eine der Linsenmasse parallel, die andere auf ihr senkrecht ist. Die Richtung der Bewegung, die jede dieser Componenten für sich unter den gegebenen Voraussetzungen einem beliebigen Punkte der Oberfläche ertheilen kann, ist derjenigen, welche die zu ihr gehörige andere Componente hervorzubringen vermag, entgegengesetzt. Die gleichzeitige Wirkung beider resultirt demnach von dem Unterschiede der den Componenten zukommenden, auf den Rand und auf die Pole bezüglichen Kraftmomente. Bezeichnet man nun das Maass des Druckes des Kammerwassers bei Erschlaffung der Zonula mit $k$, dic nit der Achse parallele Componente dieser Kraft mit s, die auf der Achse senkrechte mit w, und nimnt man die Durchschnittslinie der Meridianebene des angenommenen Punktes mit dem Aequator als Abscissenachse, so ist

$$
\begin{aligned}
& s=k \frac{d x}{\sqrt{d y^{2}+d x^{2}}}= \\
& w=k \frac{d y}{\sqrt{d y^{2}+d x^{2}}}
\end{aligned}
$$

Bezeichnet man ferner die quadrirte Oberfläche der Linse mit P, so ist die Summe des dieser Oberfläche zukommenden Druckes in der Richtung der s CompeArchiv fiir Ophthalmologie. Ix. $d$. 
tente $\int \mathrm{sdP}$, und in der Richtung der w Componente $\int \mathrm{wdP}$, und da

$$
d P=2 \pi k \sqrt{d y^{2}+d x^{2}}
$$

so ist weiter

$$
\int \mathrm{sdP}=\int 2 \pi \mathrm{k} \mathrm{xdx}=\pi \mathrm{k} \mathrm{x}^{2}
$$

und

$$
\int \mathrm{wdP}=\int 2 \pi \mathrm{k} x \mathrm{dy}
$$

Der der s-Componente entsprechende Druck ist demnach unabhängig von der Gleichung der linseneryeugenden Curve und gleich demjenigen, welchen die Constante k gleichmässig auf den $\Lambda$ equator ausüben würde, während der der w-Componente entstammende Druck je nach der Besonderheit dieser Curve variirt.

Sicht man nach der gewöhnlichen Annahme die vordere Fläche der Linse als aus der Rotation einer Eillipse entstanden an, (halbe grosse Achse -- a, halbe kleine $=$ b), deren Gleichung aus dem Mittelpunkte also

$$
y^{2}=\frac{b^{2}}{a^{2}}\left(a^{2}-x^{2}\right)
$$

so ergiebt sich

$$
\frac{d y}{d x} \cdots-\frac{b}{a} \frac{\sqrt{b^{2}}-y^{2}}{y}
$$

und 


$$
\begin{gathered}
\int \mathrm{wdP}=2 \pi \mathrm{k} \int \mathrm{xdy}= \\
\left.\frac{\pi \mathrm{ak}}{\mathrm{b}}\left[\mathrm{b}^{2} \operatorname{arc}\left(\cos =\frac{\mathrm{y}}{\mathrm{b}}\right)-\mathrm{y} \sqrt{\mathrm{b}^{2}-\mathrm{y}^{2}}\right]^{*}\right)
\end{gathered}
$$

Ist ferner dic hintere Fläche der Linse aus ciner Parabel entstanden, deren Abscissenachse in der Linsenachse liegt, deren Gleichung aus dem Scheitel also

$$
\mathrm{y}^{2}=\mathrm{px}
$$

(die Coordinaten sind der bequemeren Rechnung wegen im Vergleiche zu der Fillipsengleichung vertauscht) so ist

$$
\begin{gathered}
\frac{d y}{d x} \cdots \frac{p}{2 y} \\
\int \mathrm{wdP}=2 \pi \mathrm{k} \int \mathrm{ydk}= \\
\frac{4 \pi \mathrm{k}}{3 \mathrm{p}} \mathrm{y}^{3} .
\end{gathered}
$$

Diese Ergebnisse auf eine den Messungsmitteln entsprechende Normalellipse $\left(\mathrm{a}==2^{\prime \prime \prime}, \mathrm{b}==1^{\prime \prime \prime}\right)$ und Parabel $\left(\mathrm{p}==4^{\prime \prime \prime}\right)$ bezogen, liefern folgende Werthe:

$$
\begin{aligned}
& \text { *) } x d y=-\frac{a}{b} \sqrt{b^{2}-y^{2}} d y \\
& \mathrm{~b}^{2}-\mathrm{y}^{2}=\mathrm{y}^{2} \mathrm{z}^{2} \\
& d y=-\frac{b z d z}{\left(z^{2}+1\right)^{3 ! 2}} \\
& -\frac{a}{b} \sqrt{b^{2}-y^{2}} d y=a b \begin{array}{c}
z^{2} d z \\
\left(z^{2}+1\right)^{2}
\end{array} \\
& \frac{\mathrm{z}^{2} \mathrm{dz}}{\left(\mathrm{z}^{2}+1\right)^{2}}=-\frac{d \mathrm{z}}{\left(\mathrm{z}^{2}+1\right)^{2}}+\frac{d z}{\mathrm{z}^{2}+1} \\
& \int \frac{d z}{\left.z^{2}+1\right)^{2}}=1 / 2 \frac{z}{z^{2}} \frac{1}{1}+1 / 2 \int \frac{d z}{z^{2}+1} \\
& \int \frac{z^{2} d z}{\left(z^{2}+1\right)^{2}}=-1 / 2 \frac{z}{z^{2}+1}+1 / 2 \text { arc }(\operatorname{tang}=z) \\
& \int 2 \pi \mathrm{kab} \frac{\mathrm{z}^{2} \mathrm{dz}}{\left(\mathrm{z}^{2}+1\right)^{2}}=\pi \mathrm{kab}\left[\operatorname{arc}(\operatorname{tang}=\mathrm{z})-\frac{\mathrm{z}}{\mathrm{z}^{2}+1}\right] \\
& 2 \pi k \int x d y=\frac{\pi a k}{b}\left[b^{2} \operatorname{arc}\left(\cos =\frac{y}{b}\right)-y \sqrt{b^{2}-y^{2}}\right]
\end{aligned}
$$


1. Fillipse

$$
\begin{aligned}
& \int \mathrm{sdP}=4 \pi \mathrm{k}\left(1-\mathrm{y}^{2}\right) \\
& \int \mathrm{wdP}=2 \pi \mathrm{k}\left[\operatorname{arc}(\cos =\mathrm{y})-\mathrm{y} \sqrt{1-\mathrm{y}^{2}}\right] \\
& \int \mathrm{sdP}=\pi \mathrm{ky}^{2} \\
& \quad \text { Parabel } \\
& \int \mathrm{wdP}=\pi \mathrm{k}-\mathrm{y}^{3} .
\end{aligned}
$$

Die Insertion des vorderen und des hinteren Blattes der Zonula der Absbisse der Ellipse 1,8“" (Ordinate -$\left.0,436 . .^{\prime \prime}\right)$ entsprechend und kreisförmig genommen, sind
A. $\quad \begin{aligned} & y=1 \\ & y=0,436 . .\end{aligned} \quad \mathrm{sdP}=10,178 . . \mathrm{k}$
B. $/ \begin{aligned} & y=1 \\ & y=0,436 . .\end{aligned} \quad$ wdP $=4,571 . . k$

die Gesammtdrucke der respectiven Componenten auf die Flache der Linse, welche von dem Kammerwasser beeintlusst wird,

$$
\begin{aligned}
\text { C. } \quad \begin{aligned}
\mathrm{y} & =0,436 . . \\
\mathrm{y} & =0 \\
\mathrm{sdl} & =2,388 . . \mathrm{k} \\
\text { D. } / \mathrm{y} & =0,436 . . \\
\mathrm{y} & =0
\end{aligned} \text { wdP }-5,298 . . \mathrm{k}
\end{aligned}
$$

dieselben relativen Druckmengen für die zwischen der vorderen Insertion der Zonula und dem Rande gelegenen Theile des Petit'schen Kanales, und

$$
\begin{aligned}
& \text { E. } \int_{\mathrm{y}=1,8}^{\mathrm{y}=2} \text { sdP }=2,388 . . \mathrm{k}=\mathrm{C} \\
& \text { F. } \int \begin{array}{l}
\mathrm{y}=2 \\
\mathrm{y}=1,8
\end{array} \text { wdP }=2,270 . . \mathrm{k}
\end{aligned}
$$

die gleichen für den zwischen dem Rande und der hinteren Insertion der Zonula gelegenen Zheil. 
Im 7ustande der Jirschlaffung der Zonula ist. ler in der Achsenrichtung ühcrwiegende Druck, unter welchem die Linse steht.

$$
\alpha(\mathrm{A}+\mathrm{C} \therefore \mathrm{E})-\beta(\mathrm{B}+\mathrm{D}+\mathrm{F})
$$

wenn $\alpha$ den Aequatorialdurchmesser und $\beta$ dic $\Lambda$ chse der Linse bedeuten, weil für jeden Punkt der Fläche mit der Achse parallele Kräfte des Durchmessers des Aequators, auf ihr senkrechte dor Achse, als constanter Factoren zur Vervollständigung ihres Kraftmomentes in Beziehung auf den Pand und auf die Pole bedürfen.

Nach den bisherigen Maassen und $\alpha=4^{\prime \prime \prime}, \beta=2^{\prime \prime \prime}$, ist dieser I)ruck

$$
\text { G. } \begin{aligned}
& 4(\mathrm{~A} \vdash \mathrm{C}+\mathrm{E})-2(\mathrm{~B}+\mathrm{D}+\mathrm{H}) \\
& =35,538 . \mathrm{k}
\end{aligned}
$$

Wird dagegen die 7onula durch Muskelthätigkeit gespannt, und der Druck des Kanmerwassers dadurch um nk verstärkt, der Inruck im Petit'schen Kanal um cbensoviel vermindert, so betrügt dieser Druck nunmehr

$$
\text { H. } \begin{aligned}
2 & {[(2 \mathrm{~A}-\mathrm{B})(\mathrm{L}+\mathrm{n})+(2 \mathrm{C}-\mathrm{D}-2 \mathrm{~L}-\mathrm{F})(\mathbf{1} \mathrm{n})] } \\
& =35,538 \ldots \mathrm{k}-27,602 . . \mathrm{nk}
\end{aligned}
$$

und der Lrfolg ist derselbe, als ob die Linse in der $\Lambda$ chsenrichtung mit ciner Kraft zusammengedrückt würde, welche dem Cinterschiede $\mathrm{H}-\mathrm{G}$ oder

$$
\begin{aligned}
& 2 n(2 \Lambda+1)+\mathrm{l}-\mathrm{B}-2 \mathrm{C}-2 \mathrm{E})- \\
& 27,602 . \mathrm{nk}
\end{aligned}
$$

gleich ist.

Diese Resultirende ist in der Wirlklichkeit um ein Namhaftes grösser, weil unter Anderen die hintere Linsen erzeugende Curve in der Aequatorialgegend von der Parabel bedeutend abweicht, so dass am Aequator ihre Tangente senkrecht auf diesem steht, woraus folgt, dass F merklich grösser wird, während $\mathrm{F}$ constant bleibt, und weil die berechneten Grössen nur fïr den Anfang der 
Bewegung gelten, sobald eine solche aber eingetreten ist, alle zu Gunsten einer Vergrösserung der Differenz variiren.

Das geometrische Verhältniss der beiden Kräfte $\frac{\mathrm{H}}{\mathrm{G}}$ - schliesslich ist $1 \dashv 0,777 \ldots \mathrm{n}$. 